\title{
Precise intraoperative sentinel lymph node biopsies guided by lymphatic drainage in breast cancer
}

\author{
Xiaoyan Li ${ }^{1}$, Sisi Chen ${ }^{1}$, Liyu Jiang ${ }^{1}$, Xiaoli Kong ${ }^{1}$, Tingting $\mathrm{Ma}^{1}$, Hong $\mathrm{Xu}^{2}$ and \\ Qifeng Yang ${ }^{1,3}$ \\ ${ }^{1}$ Department of Breast Surgery, Qilu Hospital, Shandong University, Jinan, Shandong 250012, P.R. China \\ ${ }^{2}$ Department of Breast Surgery, General Hospital of Chinese Armed Police Force, Beijing 100039, P.R. China \\ ${ }^{3}$ Department of Pathology Tissue Bank, Qilu Hospital, Shandong University, Jinan, Shandong 250012, P.R. China \\ Correspondence to: Qifeng Yang, email: qifengy_sdu@163.com \\ Hong XU, email: Sing1972@sina.com
}

Keywords: precise sentinel lymph node biopsy, trSLNs, breast cancer

Received: February 13, $2017 \quad$ Accepted: May 29, $2017 \quad$ Published: June 27, 2017

Copyright: Li et al. This is an open-access article distributed under the terms of the Creative Commons Attribution License 3.0 (CC BY 3.0), which permits unrestricted use, distribution, and reproduction in any medium, provided the original author and source are credited.

\section{ABSTRACT}

The purpose of this study was to present a novel surgical method for intraoperative precise sentinel lymph node biopsy (SLNB) and to determine its clinical efficacy and sensitivity in breast cancer patients. The sentinel lymph nodes (SLNs) were preoperatively evaluated by axillary ultrasound. The intraoperative detection of SLNs was guided by lymphatic drainage pathway. The lymphatic vessels and SLNs were visualized. During operation, we searched for all the true SLNs (trSLNs), paraSLNs (paSLNs) and post-SLNs (poSLNs) followed lymphatic drainage ducts. After precisely locating the lymphatic channels and lymph node, all the lymph nodes that firstly receive lymphatic drainage are designated as trSLNs. We precisely distinguished the trSLNs, paSLNs and poSLNs. We found the average number of trSLNs ranged from 1 to 6 . In addition, we assessed the novel technique in a total of 125 breast cancer patients. trSLNs were successfully identified in all patients (detection rate: $100 \%$ ). The accuracy of trSLNs is $\mathbf{9 9 . 2 \%}$. Data from our study strongly suggest that our method is a feasible and effective for the detection of precise trSLNs in breast cancer with real-time observations. (ClinicalTrials.gov number, NCT02651142).

\section{INTRODUCTION}

Sentinel lymph nodes (SLN) are the first lymph nodes that receive lymphatic drainage. Cancer cells are mainly spread through the lymphatic system in early stage of breast cancer. Regional lymph node status is one of the strongest prognostic factors for primary breast cancer. Therefore it is important to exactly identify positive lymph nodes for axillary management because falsenegative results will lead to inaccurate assessment of regional lymph node and inadequate therapeutic strategies. Axillary lymph node dissection has been used to evaluate lymph node status. However, it appears correlated with higher morbidity of lymphedema, arm pain and stiffness, seroma formation. At present sentinel lymph nodes biopsy (SLNB) has been used successfully for axillary evaluation in breast cancer and it's considered the standard method for early-stage breast cancer without clinically or radiologically axillary lymph node metastases $[1,2]$. The American Society of Clinical Oncology recommend the minimum sentinel node identification rate is $85 \%$ and the false-negative rate is less than $5 \%$ before abandoning the axillary dissection [3]. Studies shown that the dual technique involving injection of radioisotope and blue dye into either the interstitial breast tissue around the tumor or the periareolar tissue are common methods for SLN biopsy [4]. Also it is possible to use each one independently. Though many new techniques, such as Indocyanine green (ICG), have been employed for identifying SLN $[5,6]$, the specificity and risk of false-negative rate in mapping SLNs are still the main concern. 
Today there are still many debates involving SLN biopsy. One is the exact number of SLNs for patients. Studies showed that patients may have different types of sentinel lymph channels and different numbers of SLNs $[7,8]$. As the drainage of lymph nodes are in multiple directions from the primary tumor, the type of sentinel lymph channels could affect the detection rate of SLN biopsy. This could partly explain that the false-negative rate of SLN failed to be achieved even for experienced surgeons. Previous studies have found that the number of SLNs removed has a statistical effect on the false-negative rate $[9,10]$. In many studies, lymph nodes that contained ICG fluorescence, radioactivity and/or blue staining were usually defined as SLNs and excised by surgeons [11-13]. Studies have reported that the SLNs ranged from 1 to 12 for patients in many countries including China, Germany, Poland, USA and Netherlands [14], and this could not be explained by races. As the number of SLNs for one patient is fixed, we probably removed more lymph nodes that are not true SLNs in order to reduce the false-negative rate clinically. The overtreatment is even more common in post-neoadjuvant chemotherapy. Boughey et al. [15] reported that removing at least two sentinel nodes could lower the false-negative rate of SLNs in post-neoadjuvant chemotherapy. The similar result was reported by Boileau and his colleges [16]. However, if only one true SLN actually exist in patients, excess axillary lymph node that are not true SLNs will be dissected. On the other hand, when the true SLNs are not fully dissected, the remaining SLNs maybe positive and the patients may have increased local recurrence and poor prognosis due to the false-negativity of SLNB. Therefore, other methods for increasing the sentinel lymph node identification rate and reducing the false-negative rate are welcome to be explored. In this study, we present a novel surgical method that enables the precise identification of trSLNs guided by lymphatic drainage pathway in breast cancer.

\section{RESULTS}

\section{Precise sentinel lymph node biopsy}

All patients received axillary ultrasound and all the possible sentinel lymph nodes were preoperative assessed and marked on the skin (Figure 1A). In our study, we injected methylene blue into the periareolar tissue in multiple deposits directly before surgery. Five minutes later, ICG were injected in the same position. Then the surgical field was illuminated using a near-infrared illumination system for ICG lymphography (Ming De Fluorescence Imaging System). As ICG fluorescence illumination can be captured to a depth of less than 2 $\mathrm{mm}$, lymph flow over the breast skin surface and in the superficial tissues can be visualized. Guided by the ICG fluorescence real-time dynamic imaging system, the shiny lymphatic vessels could be observed transcutaneously in real time on the monitor screen (Figure 1A and 1B). We next marked the lymphatic drainage pathways with a pen so that we don't disrupt the lymphatic channels during surgery (Figure 1C). Represented images of marked lymphatic drainage pathways guided by ICG fluorescence were shown in Figure 2. As the whole lymphatic vessels were marked on skin, we incised the skin and fully dissect out the lymphatic vessels guided by both fluorescence and blue stained lymphatic duct. The lymph nodes that accumulated methylene blue stained blue could be observed with the naked eye while that accumulated ICG appeared as shiny fluorescent spots. After incision of skin, the lymphatic drainage pathways and SLNs were observed apparently. The lymph nodes were stained by both ICG and methylene blue while others exhibit only green staining or blue staining. Then we searched for SLNs directly guided by lymphatic drainage pathway and exactly dissected out the lymphatic vessels and lymph nodes. In clinical practice, we found that the time interval after injection methylene blue and ICG can affect the staining of lymph nodes. If the time is not enough, the SLNs may not fully identified especially for SLNs with more than one lymphatic ducts. Otherwise, the non-SLNs were also stained. Our time interval was about 15 minutes after injection ICG. After precisely locating the lymphatic channels and lymph node, all the green staining and blue staining lymph nodes that firstly receive lymphatic drainage are designated as trSLNs. Our finding showed that different trSLNs had different number of input lymphatic ducts. Some had one input lymphatic duct while others had two or more ducts (Figure 3). What's more, we found that some lymph nodes that directly followed the trSLNs could be blue stained and had fluorescence. We defined that lymph node as post-SLNs (poSLNs) (Figure 4). If we did not dissected out and marked all the lymphatic drainage pathways. We may remove the poSLNs as trSLNs. In addition, we found many swollen lymph nodes closed to trSLNs and we defined these as para-SLNs (paSLNs). They weren't stained neither by fluorescence nor methylene blue dye (Figure 5). These maybe also be removed by surgeons clinically. If so the SLNB for these patients should be more carefully and remove the trSLNs without poSLNs or paSLNs in clinical practice.

\section{Evaluation on clinical application of SLNB}

In order to determine the clinical efficacy and sensitivity of our method in breast cancer patients, 125 breast cancer patients were enrolled in our clinical trials for verification. The patients were diagnosed as invasive ductal carcinoma or ductal carcinoma in situ or invasive lobular carcinoma. Axillary lymph node dissection was performed in all the patients so we could precisely evaluation the status of locoreginal lymph node. The average age of patients was 49.47 years (range, 31-78 years). None 

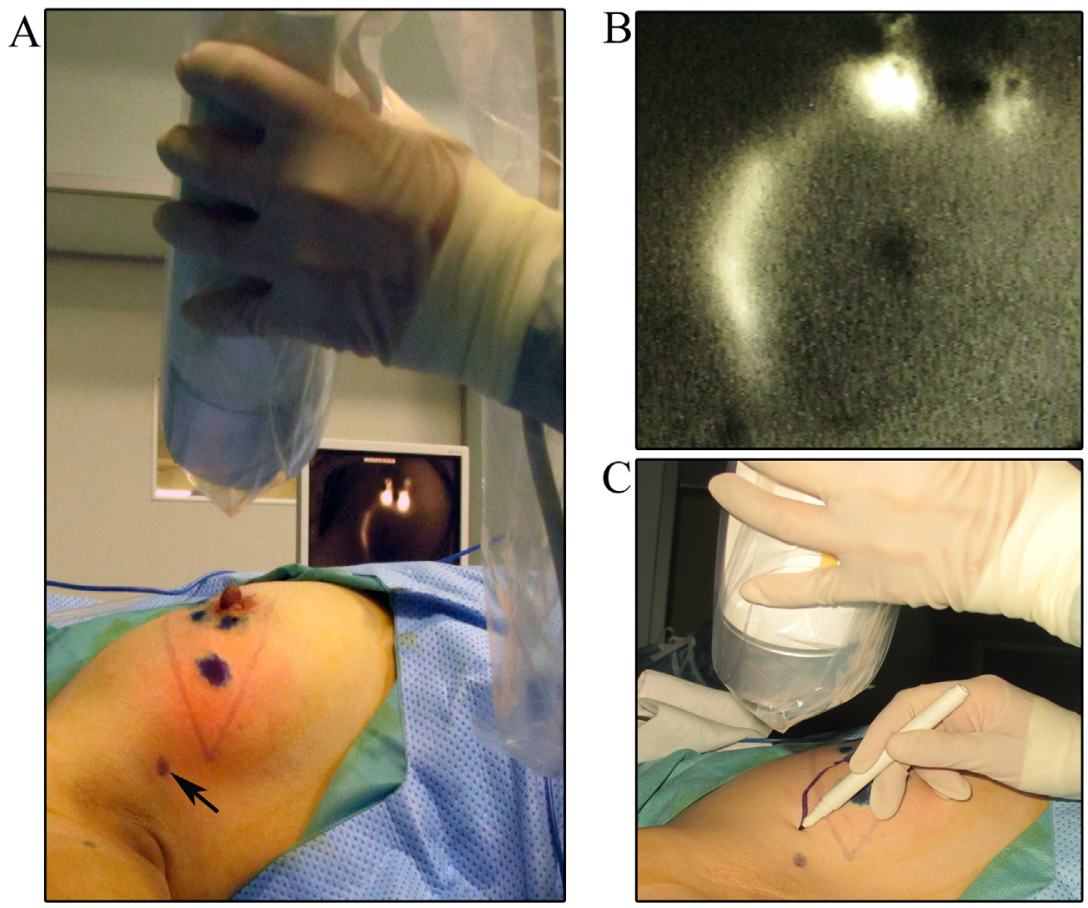

Figure 1: Marking the whole lymphatic vessels guided by ICG fluorescence. (A) The indocyanine green fluorescence real-time dynamic imaging system. Black arrows were the possible SLNs under preoperatively axillary ultrasound assessment. (B) ICG fluorescence image of lymphatic vessels visualized in real time on the monitor screen of the dynamic imaging system. (C) Drawing the lymphatic drainage pathways guided by ICG fluorescence.
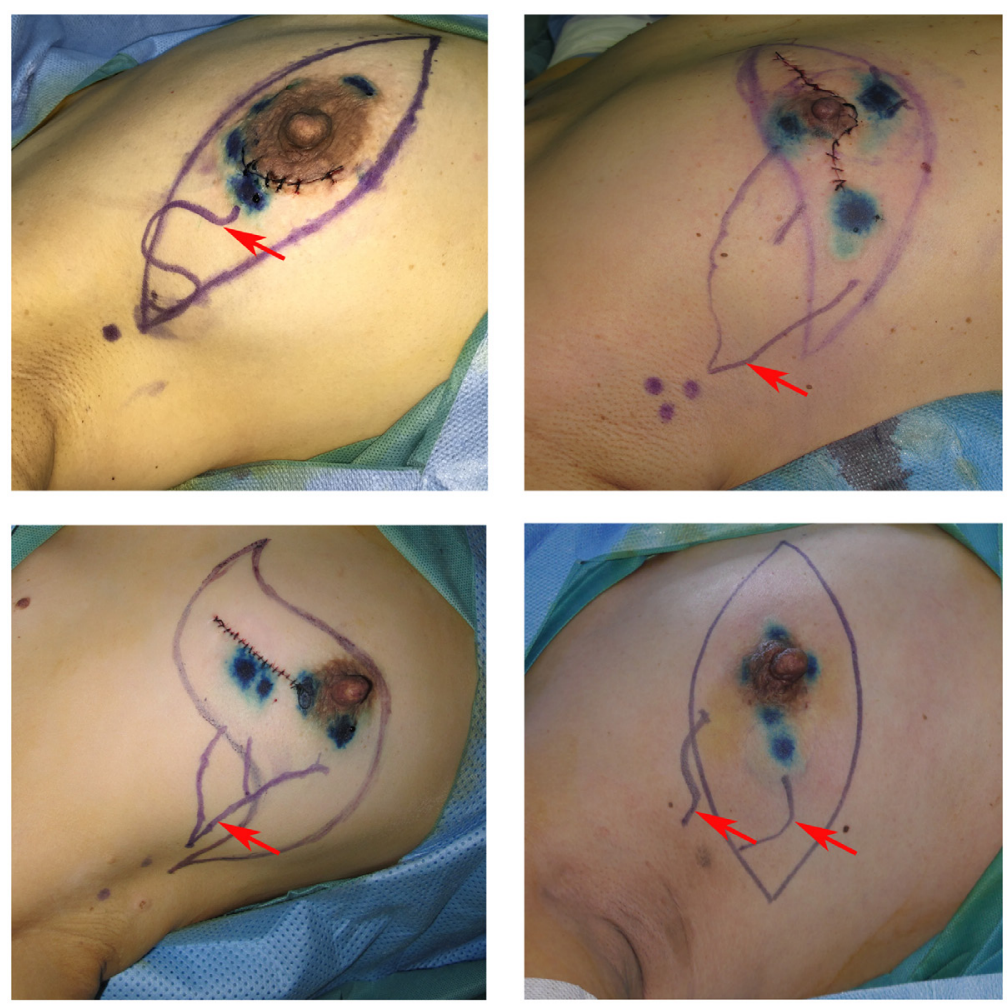

Figure 2: Represented images of marked lymphatic drainage pathways guided by ICG fluorescence. Red arrows were the lymphatic channels. 

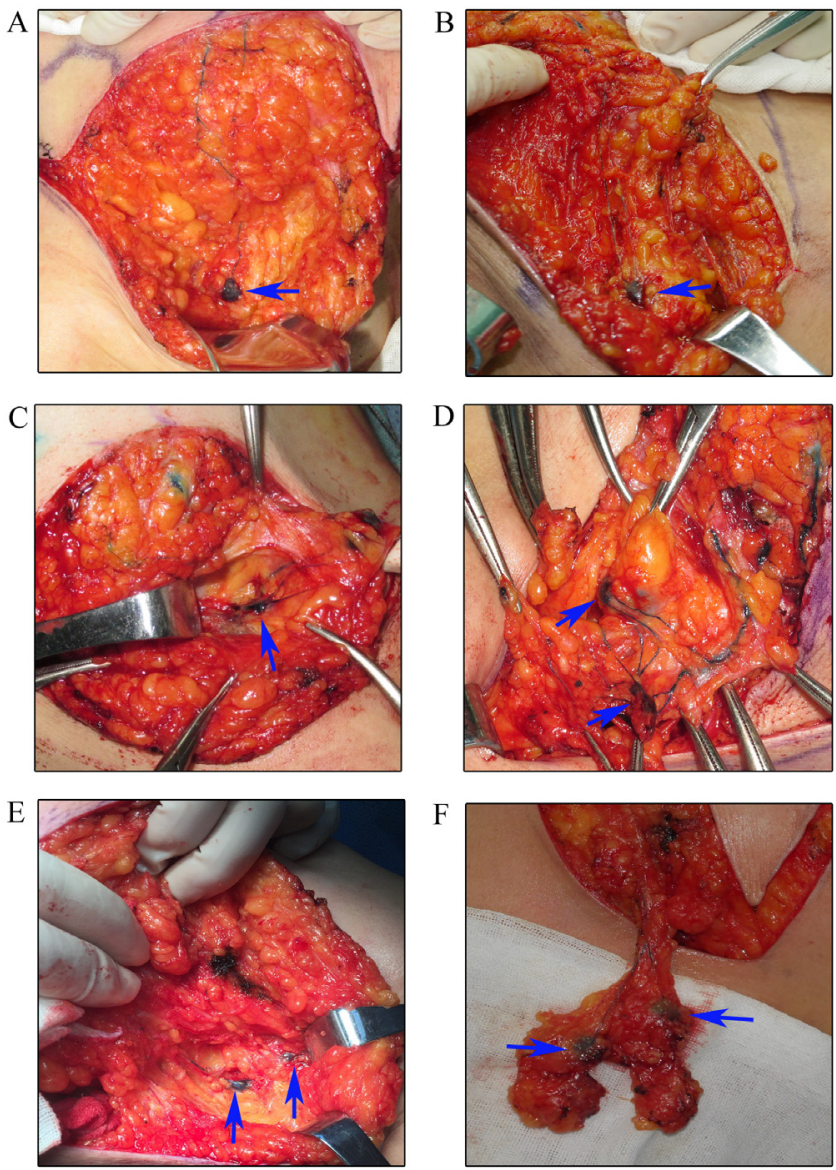

Figure 3: Identification the trSLNs with various input lymphatic ducts. (A) trSLN with one input lymphatic duct. (B) trSLN with one input lymphatic duct. (C) trSLN with three input lymphatic ducts. (D) trSLN with multiple input lymphatic ducts. (E) SLNs with two parallel input lymphatic ducts. (F) trSLNs with bifurcated input lymphatic vessels. Blue arrows were SLNs.
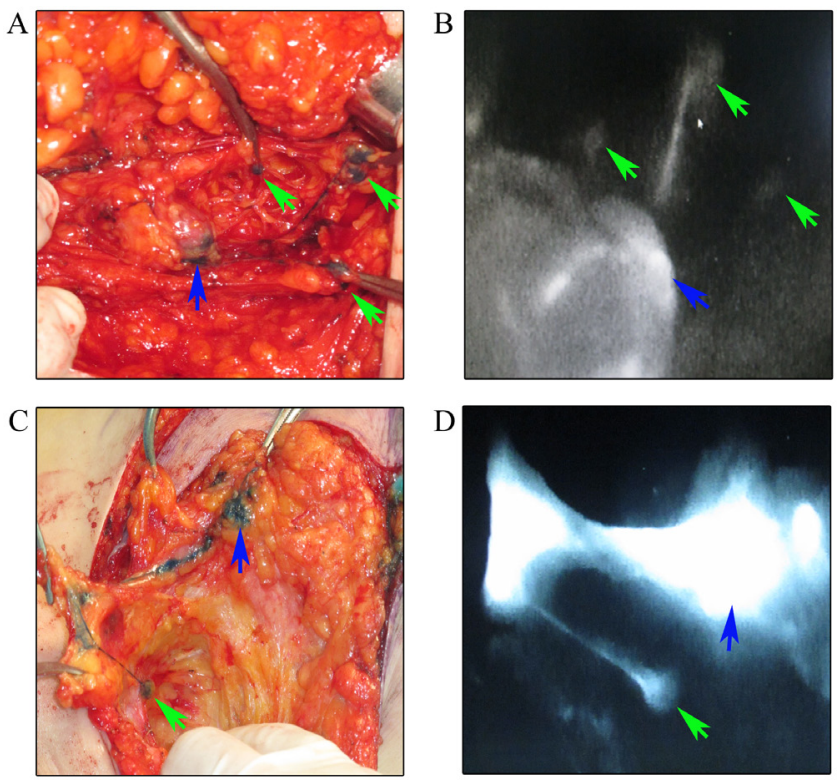

Figure 4: Identification the trSLNs with poSLNs. (A) Dissection the whole lymphatic vessels and trSLN with three poSLNs. (B) The shiny fluorescent images of A on the monitor screen of the dynamic imaging system. (C) Dissection the whole lymphatic vessels and trSLN with one poSLN. (D) The shiny fluorescent images of C on the monitor screen of the dynamic imaging system. Blue arrows were trSLNs and green arrows were poSLNs. 
of the patients experienced severe adverse reactions or complications that were related to the intraoperative injection of ICG combined with methylene blue. It's exciting that we identified all the trSLNs by our novel surgical navigation method (detection rate: $100 \%$ ). A total of 42 patients have 1 trSLN, 41 patients have 2 trSLNs, 19 patients have 3 trSLNs, 14 patients have 4 trSLNs, 4 patients have 5 trSLNs, 5 patients have 6 trSLNs. The average number of trSLNs was 2.3 (range 1-6 trSLNs) in all patients. In our practice, about $45.6 \%(57 / 125)$ patients had positive SLNs while the other 68 were negative. A total of 57 patients were trSLNs positive. 1 case was ductal carcinoma in situ with microinvasion, 1 case was invasive lobular carcinoma and 55 cases were invasive ductal carcinoma. Among these trSLNs positive 57 patients, 31 patients have no additional metastatic axillary lymph node. However, in the 58 trSLNs negative patients, only one had metastatic lymph node in the dissected axillary lymph node. The false negative rate is $1.7 \%$ in our study. The accuracy of our data is $99.2 \%$. By further analyzing the data, we found this patient had four tumors in breast. Therefore, the lymphatic vessels may be disrupted when excision the tumors for biopsy. The positive lymph node probably was a true SLN that we did not identify. This should be further confirmed in the following study.

\section{DISCUSSION}

SLNB has been adopted as a standard method for axillary nodal staging by most surgeons [17]. At present, a blue dye or a radioactive tracer, and/or a combination of these two methods are the most performed techniques for SLN mapping and identification [13, 18]. Large randomized trials showed that the overall accuracy SLNs was $93 \%$ to $97 \%$ and a false negative rate was about 9.8\% [19-21]. The SLN identification rate is lower and false negative rate is higher in patients receiving postneoadjuvant chemotherapy. Clinical trials reported a higher false negative rate when only one SLN was found [22]. Kennedy reported that removal of two sentinel nodes significantly reduced the false-negative rate compared with removal of one node [23]. Other studies suggested that four might be an optimal threshold number of SLNs to be removed $[24,25]$. Aoyama and Chi showed that the detection rate was $100 \%$ when they removed $1 \sim 12$ SLNs $[26,27]$.
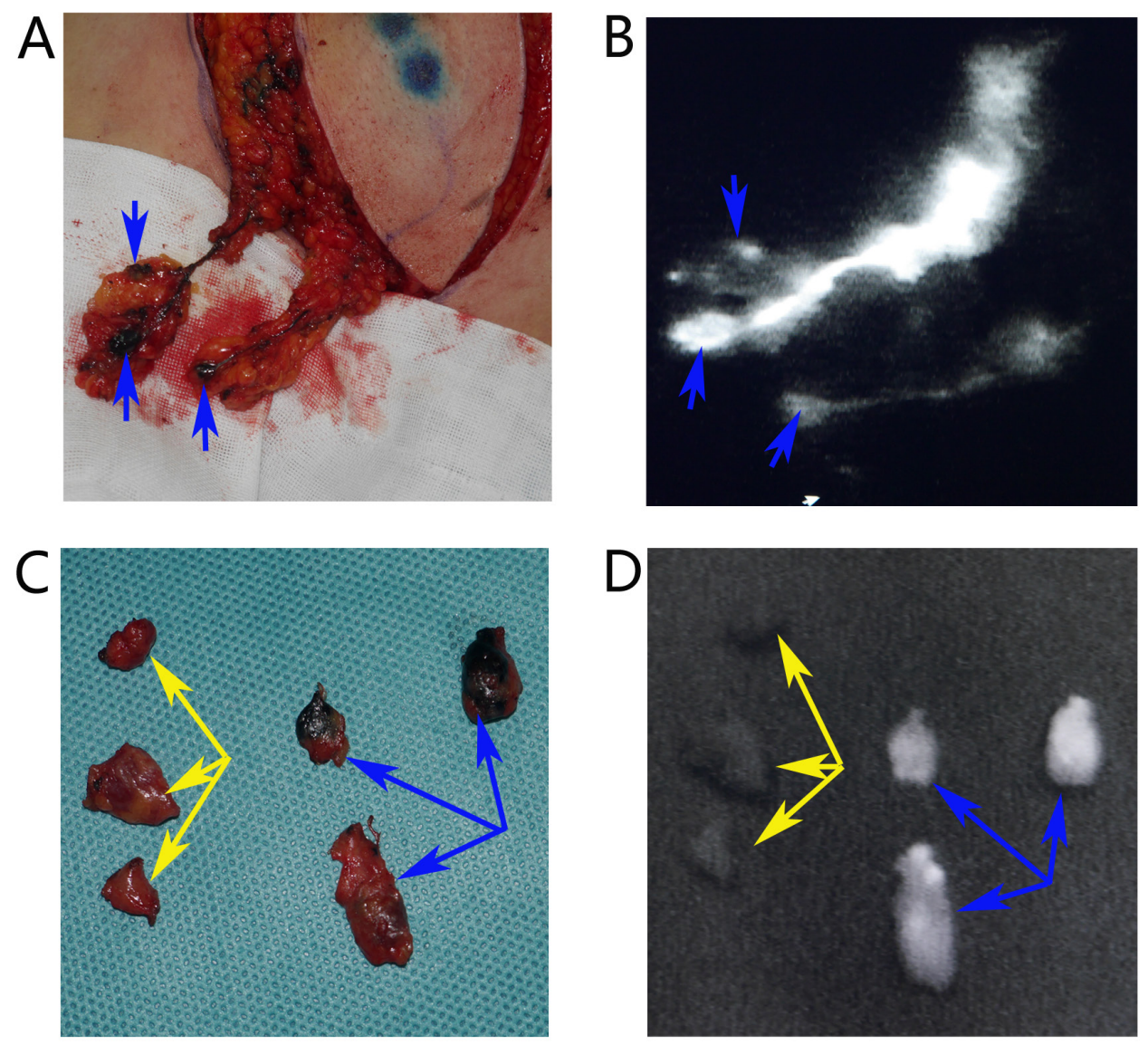

Figure 5: Identification the trSLNs with paSLNs. (A) Dissection the whole lymphatic vessels and trSLNs. (B) The shiny fluorescent images of A on the monitor screen of the dynamic imaging system. (C) All the resected trSLNs and paSLNs. (D) The shiny fluorescence images of all trSLNs and paSLNs. Blue arrows were trSLNs and yellow arrows were paSLNs. 
Table 1: Clinicopathological features of the patients and tumors

\begin{tabular}{|c|c|}
\hline Characteristics & Number \\
\hline \multicolumn{2}{|l|}{ Age } \\
\hline$\leq 45$ & 48 \\
\hline$>45$ & 77 \\
\hline \multicolumn{2}{|l|}{ Histologic subtype } \\
\hline Invasive ductal carcinoma & 109 \\
\hline Invasive Lobular carcinoma & 2 \\
\hline Others & 14 \\
\hline \multicolumn{2}{|l|}{ Number of tumors } \\
\hline 1 & 118 \\
\hline 2 & 3 \\
\hline 3 & 3 \\
\hline 4 & 1 \\
\hline \multicolumn{2}{|l|}{ Tumor size } \\
\hline$\leq 2 \mathrm{~cm}$ & 68 \\
\hline$>2 \mathrm{~cm}$ & 57 \\
\hline \multicolumn{2}{|l|}{ ER status } \\
\hline Negative & 36 \\
\hline Positive & 89 \\
\hline \multicolumn{2}{|l|}{ PR status } \\
\hline Negative & 40 \\
\hline Positive & 85 \\
\hline \multicolumn{2}{|l|}{ Her-2 status } \\
\hline Negative & 76 \\
\hline Positive & 49 \\
\hline
\end{tabular}

ER: Estrogen Receptor; PR: Progesterone Receptor;

Her-2: human epidermalgrowth factor receptor-2.

How many SLNs should be resected? As SLNs are the definition of anatomy and patients have different types of sentinel lymph channels and different numbers of SLNs, so in our opinion we couldn't define the number of SLNs that should be removed. On the contrary, we should dissect all the true SLNs. Though alternative techniques for increasing the SLNs accuracy rate and reducing the false-negative rate need to be developed, the most important is the method used to identify the true SLNs in clinical practice. As we all know axillary ultrasound can identify the status of axillary lymph nodes based on sizes and morphology of the lymph nodes and it's considered as a noninvasive technique for staging of the axilla $[28,29]$. In the present study, we firstly evaluate the status by axillary ultrasound, and we localized the suspected metastatic lymph node. Then we did precise SLNB followed by lymphatic drainage pathway with the help of ICG fluorescence and methylene blue dye. The surgical procedure could be used to precisely locate SLNs and the lymphatic vessels. Then we exactly dissect out all the lymphatic vessels and SLNs for patients receiving mastectomy. For patients receiving breast conservation surgery, the ICG fluorescence image of lymphatic vessels can be visualized on the skin surface, and the location of the skin incision for the detection of SLNs can be designed intraoperatively at the end of the lymphatic vessels. Then the lymphatic drainage ducts could be dissected out. And we removed the trSLNs guided by fluorescence and blue 
stained lymph nodes. What's more important, we could distinguish between the trSLNs, paSLNs and poSLNs. In many studies, surgeons may remove the paSLNs and poSLNs and defined as trSLNs. By using our method, we could easily and exactly identify the trSLNs rather than paSLNs and poSLNs. We found that the average number of trSLNs ranged from 1 to 6 in breast cancer patients. In addition, our clinical practice further demonstrated the feasibility and accuracy of our method for precise SLNB in breast cancer. Our data suggest that the novel technique could not only provide the accurate location of trSLNs and lymphatic mapping, but it also be used to successfully remove the trSLNs with a high sensitivity and accuracy. Therefore, this method potentially provides an alternative for the exact SLNB in breast cancer. However, our present study still had some limitations. First, the number of patients is relatively small. Second, there is no prognosis and complications information for patients. In addition, early breast cancer is treated usually by breast conserving surgery the SLNB. We should explore easier methods for identifying trSLN in these cases with small incisions. Therefore a large randomized, controlled trial is needed in the future.

In conclusion, our data have demonstrated that our method exhibited a very high sensitivity as well as lower false negative rate. Also it could provide a more accurate detection of the true SLNs clinically. With the novel method, we could easily identify the true SLNs precisely and it will provide important information to guide precise treatment decisions for breast cancer patients in the future.

\section{MATERIALS AND METHODS}

\section{Patients}

A total of 125 breast cancer patients were enrolled. All the patients were received SLNB firstly, and then followed by axillary lymph node dissection (ALND) between September 2015 and Auguest 2016 in Qiu Hospital of Shandong University. Inclusion criteria were a histopathological diagnosis of breast cancer, and an indication for mastectomy or breast conservation therapy without a clinically positive lymph node status. Exclusion Criteria were fine needle aspiration cytology was positive patient. The detailed clinical data and tumor characteristics are summarized in Table 1. After precisely locating the lymphatic channels and lymph node, all the lymph nodes that firstly receive lymphatic drainage are designated as trSLNs. The lymph nodes that directly followed the trSLNs were defined as post-SLNs (poSLNs). These swollen axillary lymph nodes around the trSLNs were defined as para-sentinel lymph nodes (para-SLNs). Written informed consent was obtained and our project was approved by Ethics Committee on Scientific Research of Shandong University Qilu Hospital. A histopathological assessment of the resection specimens were conducted postoperatively.

\section{Statistical analyses}

The accuracy and false-negative rate were calculated by comparing the results of the SLNB and the histopathology of the resection specimens in axillary lymph node dissection. False negative rate $=$ number of false negative SLNs / (true positive + false negative nodes $) \times 100$. Accuracy $=($ true positive + true negative nodes)/ total nodes x 100 .

\section{ACKNOWLEDGMENTS AND FUNDINGS}

This work was supported by the National Natural Science Foundation of China (No.81672613; No.81602329), Key Research and Development Program of Shandong Province (No.2015GSF118093; No.2016GGE2775), Shandong Science and Technology Development Plan (2016CYJS01A02) and Special Support Plan for National High Level Talents ("Ten Thousand Talents Program”) to Qifeng Yang.

\section{CONFLICTS OF INTEREST}

The authors declare no conflicts of interest.

\section{REFERENCES}

1. Lyman GH, Temin S, Edge SB, Newman LA, Turner RR, Weaver DL, Benson AB, 3rd, Bosserman LD, Burstein HJ, Cody H, 3rd, Hayman J, Perkins CL, Podoloff DA and Giuliano AE. Sentinel lymph node biopsy for patients with early-stage breast cancer: American Society of Clinical Oncology clinical practice guideline update. J Clin Oncol. 2014; 32:1365-1383.

2. Vuthaluru S and Srivastava A. Axillary vs sentinel lymph node dissection for invasive breast cancer. JAMA. 2011; 305:2290; author reply 2290-2291.

3. Lyman GH, Giuliano AE, Somerfield MR, Benson AB, 3rd, Bodurka DC, Burstein HJ, Cochran AJ, Cody HS, 3rd, Edge SB, Galper S, Hayman JA, Kim TY, Perkins CL, Podoloff DA, Sivasubramaniam VH, Turner RR, et al. American Society of Clinical Oncology guideline recommendations for sentinel lymph node biopsy in early-stage breast cancer. J Clin Oncol. 2005; 23:7703-7720.

4. Zavagno G, De Salvo GL, Scalco G, Bozza F, Barutta L, Del Bianco P, Renier M, Racano C, Carraro P and Nitti D. A Randomized clinical trial on sentinel lymph node biopsy versus axillary lymph node dissection in breast cancer: results of the Sentinella/GIVOM trial. Ann Surg. 2008; 247:207-213.

5. Verbeek FP, Troyan SL, Mieog JS, Liefers GJ, Moffitt LA, Rosenberg M, Hirshfield-Bartek J, Gioux S, van de Velde CJ, 
Vahrmeijer AL and Frangioni JV. Near-infrared fluorescence sentinel lymph node mapping in breast cancer: a multicenter experience. Breast Cancer Res Treat. 2014; 143:333-342.

6. Ahmed M, Purushotham AD and Douek M. Novel techniques for sentinel lymph node biopsy in breast cancer: a systematic review. Lancet Oncol. 2014; 15:e351-362.

7. Wang M, Zhou W, Zhao Y, Xia T, Zha X, Ding Q, Liu X, Ling L, Chen L and Wang S. A novel finding of sentinel lymphatic channels in early stage breast cancer patients: which may influence detection rate and false-negative rate of sentinel lymph node biopsy. PLoS One. 2012; 7:e51226.

8. Suami H, Pan WR, Mann GB and Taylor GI. The lymphatic anatomy of the breast and its implications for sentinel lymph node biopsy: a human cadaver study. Ann Surg Oncol. 2008; 15:863-871.

9. Krag DN, Anderson SJ, Julian TB, Brown AM, Harlow SP, Ashikaga T, Weaver DL, Miller BJ, Jalovec LM, Frazier TG, Noyes RD, Robidoux A, Scarth HM, Mammolito DM, McCready DR, Mamounas EP, et al. Technical outcomes of sentinel-lymph-node resection and conventional axillarylymph-node dissection in patients with clinically nodenegative breast cancer: results from the NSABP B-32 randomised phase III trial. Lancet Oncol. 2007; 8:881-888.

10. Goyal A, Newcombe RG, Chhabra A and Mansel RE. Factors affecting failed localisation and false-negative rates of sentinel node biopsy in breast cancer--results of the ALMANAC validation phase. Breast Cancer Res Treat. 2006; 99:203-208.

11. Sugie T, Kinoshita T, Masuda N, Sawada T, Yamauchi A, Kuroi K, Taguchi T, Bando H, Yamashiro H, Lee T, Shinkura N, Kato H, Ikeda T, Yoshimura K, Ueyama H and Toi M. Evaluation of the Clinical Utility of the ICG Fluorescence Method Compared with the Radioisotope Method for Sentinel Lymph Node Biopsy in Breast Cancer. Ann Surg Oncol. 2016; 23:44-50.

12. Toh U, Iwakuma $\mathrm{N}$, Mishima $\mathrm{M}$, Okabe $\mathrm{M}$, Nakagawa $\mathrm{S}$ and Akagi Y. Navigation surgery for intraoperative sentinel lymph node detection using Indocyanine green (ICG) fluorescence real-time imaging in breast cancer. Breast Cancer Res Treat. 2015; 153:337-344.

13. Hirche C, Murawa D, Mohr Z, Kneif S and Hunerbein M. ICG fluorescence-guided sentinel node biopsy for axillary nodal staging in breast cancer. Breast Cancer Res Treat. 2010; 121:373-378.

14. Zhang X, Li Y, Zhou Y, Mao F, Lin Y, Guan J and Sun Q. Diagnostic Performance of Indocyanine Green-Guided Sentinel Lymph Node Biopsy in Breast Cancer: A MetaAnalysis. PLoS One. 2016; 11:e0155597.

15. Boughey JC, Ballman KV, Hunt KK, McCall LM, Mittendorf EA, Ahrendt GM, Wilke LG and Le-Petross HT. Axillary Ultrasound After Neoadjuvant Chemotherapy and Its Impact on Sentinel Lymph Node Surgery: Results From the American College of Surgeons Oncology Group Z1071 Trial (Alliance). J Clin Oncol. 2015; 33:3386-3393.
16. Boileau JF, Poirier B, Basik M, Holloway CM, Gaboury L, Sideris L, Meterissian S, Arnaout A, Brackstone M, McCready DR, Karp SE, Trop I, Lisbona A, Wright FC, Younan RJ, Provencher L, et al. Sentinel node biopsy after neoadjuvant chemotherapy in biopsy-proven node-positive breast cancer: the SN FNAC study. J Clin Oncol. 2015; 33:258-264.

17. Anderson BO and Gralow JR. Axillary vs sentinel lymph node dissection for invasive breast cancer. JAMA. 2011; 305:2290; author reply 2290-2291.

18. Schaafsma BE, Verbeek FPR, Rietbergen DDD, van der Hiel B, van der Vorst JR, Liefers GJ, Frangioni JV, van de Velde CJH, van Leeuwen FWB and Vahrmeijer AL. Clinical trial of combined radio- and fluorescence-guided sentinel lymph node biopsy in breast cancer. British Journal of Surgery. 2013; 100:1037-1044.

19. Lyman GH, Temin S, Edge SB, Newman LA, Turner RR, Weaver DL, Benson AB, 3rd, Bosserman LD, Burstein HJ, Cody H, 3rd, Hayman J, Perkins CL, Podoloff DA, Giuliano $\mathrm{AE}$ and American Society of Clinical Oncology Clinical P. Sentinel lymph node biopsy for patients with early-stage breast cancer: American Society of Clinical Oncology clinical practice guideline update. J Clin Oncol. 2014; 32:1365-1383.

20. Krag DN, Anderson SJ, Julian TB, Brown AM, Harlow SP, Ashikaga T, Weaver DL, Miller BJ, Jalovec LM, Frazier TG, Noyes RD, Robidoux A, Scarth HM, Mammolito DM, McCready DR, Mamounas EP, et al. Technical outcomes of sentinel-lymph-node resection and conventional axillarylymph-node dissection in patients with clinically nodenegative breast cancer: results from the NSABP B-32 randomised phase III trial. Lancet Oncol. 2007; 8:881-888.

21. Ahmed M, Purushotham AD, Horgan K, Klaase JM and Douek M. Meta-analysis of superficial versus deep injection of radioactive tracer and blue dye for lymphatic mapping and detection of sentinel lymph nodes in breast cancer. Br J Surg. 2015; 102:169-181.

22. Kuehn T, Bauerfeind I, Fehm T, Fleige B, Hausschild M, Helms G, Lebeau A, Liedtke C, von Minckwitz G, Nekljudova V, Schmatloch S, Schrenk P, Staebler A and Untch M. Sentinel-lymph-node biopsy in patients with breast cancer before and after neoadjuvant chemotherapy (SENTINA): a prospective, multicentre cohort study. Lancet Oncol. 2013; 14:609-618.

23. Kennedy RJ, Kollias J, Gill PG, Bochner M, Coventry BJ and Farshid G. Removal of two sentinel nodes accurately stages the axilla in breast cancer. Br J Surg. 2003; 90:1349-1353.

24. Zakaria S, Degnim AC, Kleer CG, Diehl KA, Cimmino VM, Chang AE, Newman LA and Sabel MS. Sentinel lymph node biopsy for breast cancer: how many nodes are enough? J Surg Oncol. 2007; 96:554-559.

25. Ban EJ, Lee JS, Koo JS, Park S, Kim SI and Park BW. How many sentinel lymph nodes are enough for accurate axillary staging in t1-2 breast cancer? J Breast Cancer. 2011; 14:296-300. 
26. Aoyama K, Kamio T, Ohchi T, Nishizawa M and Kameoka S. Sentinel lymph node biopsy for breast cancer patients using fluorescence navigation with indocyanine green. World J Surg Oncol. 2011; 9:157.

27. Chi C, Ye J, Ding H, He D, Huang W, Zhang GJ and Tian J. Use of indocyanine green for detecting the sentinel lymph node in breast cancer patients: from preclinical evaluation to clinical validation. PLoS One. 2013; 8:e83927.
28. Yamashita M, Hovanessian-Larsen L and Sener SF. The role of axillary ultrasound in the detection of metastases from primary breast cancers. Am J Surg. 2013; 205:242-244; discussion 244-245.

29. Tucker NS, Cyr AE, Ademuyiwa FO, Tabchy A, George K, Sharma PK, Jin LX, Sanati S, Aft R, Gao F, Margenthaler JA and Gillanders WE. Axillary Ultrasound Accurately Excludes Clinically Significant Lymph Node Disease in Patients With Early Stage Breast Cancer. Ann Surg. 2016. 